\title{
Review Article \\ mTORC1 Signaling-Activated Increases in Muscle Protein Synthesis induced by leucine and Phosphatidic Acid: A Brief Review
}

\author{
Paul Hwang and Darryn S. Willoughby*
}

Department of Health, Human Performance, and Recreation, Exercise and Biochemical Nutrition Laboratory, Baylor University, Waco, TX 76798, USA

\section{Received Date: November 13, 2014 \\ Accepted Date: January 07, 2015 \\ Published Date: January 09, 2015}

Citation: Darryn, S.W., et al. mTORC1 Signaling-Activated Increases in Muscle Protein Synthesis induced by leucine and Phosphatidic Acid: A Brief Review (2015) J Food Nutr Sci 2(1): 35-39.

Corresponding author: Darryn Willoughby, Baylor University, Department of Health, Human Performance, and Recreation, Waco, TX 76798, USA, Tel: (254) 710-3504; E-mail: darryn_willoughby@baylor.edu

Keywords: Leucine; Phosphatidic acid; mTORC1; Protein synthesis; Skeletal muscle

\begin{abstract}
The mammalian target of rapamycin (mTOR) is a signaling network that functions to regulate muscle protein synthesis (MPS). The effects of leucine and phosphatidic acid (PA) availability and growth factors such as insulin and insulin-like growth factor 1 (IGF-1) can up-regulate this signaling pathway and increase the initiation of protein translation necessary for MPS. mTORC1 is known to be translocated to the lysosome where it is subsequently activated by the Rheb and leucine-Ragulator/Rag GTPase pathways and human vacuolar protein sorting 34 (hVps34)-PA axis. The diacyl-glycerophospholipid, PA, is suggested to act as an intracellular lipid second messenger that mediates cell signaling activity. PA is synthesized by various classes of enzymes such as phospholipase D (PLD) and directly activates mTORC1 by binding to the FKBP12-rapamycin-binding (FRB) domain of mTORC1. Another alternative mechanism through which PA supposedly promotes activation of mTORC1 may also be through the extracellular regulated kinase (ERK) signaling pathway. This brief review will highlight the mechanisms of action involved in MPS due to mTORC1 activation resulting from leucine and PA availability.
\end{abstract}

\section{PI3K/Akt/mTOR Signaling and Muscle Protein Synthesis section:}

The mammalian target of rapamycin (mTOR) is an integrative signaling pathway that mediates various intracellular processes in skeletal muscle. It has been clearly established that the intracellular mTOR signaling pathway has an essential role in regulating muscle protein synthesis (MPS $)^{[1-5]}$. As can be seen in Figure 1,stimuli such as the availability of the branched-chain amino acid (BCAA) leucine, the diacyl-glycerophospholipid, phosphatidic acid (PA), and heightened mitogenic activity imposed by insulin and insulin-like growth factor 1 (IGF-1) can activate mTOR signaling. Increased mTOR signaling can then lead to increases in the initiation of protein translation and subsequent MPS. Within the mTOR signaling pathway, insulin and IGF-1 bind to their respective receptors, which then triggers the activation of various kinases such as phosphatidylinositol-3 kinase $(\mathrm{PI} 3 \mathrm{~K})^{[6]}$. The activated PI3K then phosphorylates and forms the membrane phospholipid, phosphatidylinositol- 3,4,5-triphosphate (PIP3), which opens a lipid-binding membrane site for effector protein kinase $\mathrm{B}(\mathrm{Akt})^{[6]}$. Akt signaling has a range of cellular functions such as the modulation of glycolysis and the regulation of both phosphofructokinase (PFK) activity and gene transcription through the inactivation of the fork head box $\mathrm{O}$ (FOXO) transcription factors; these factors function within skeletal muscle atrophy-related signaling pathways ${ }^{[1,7]}$.

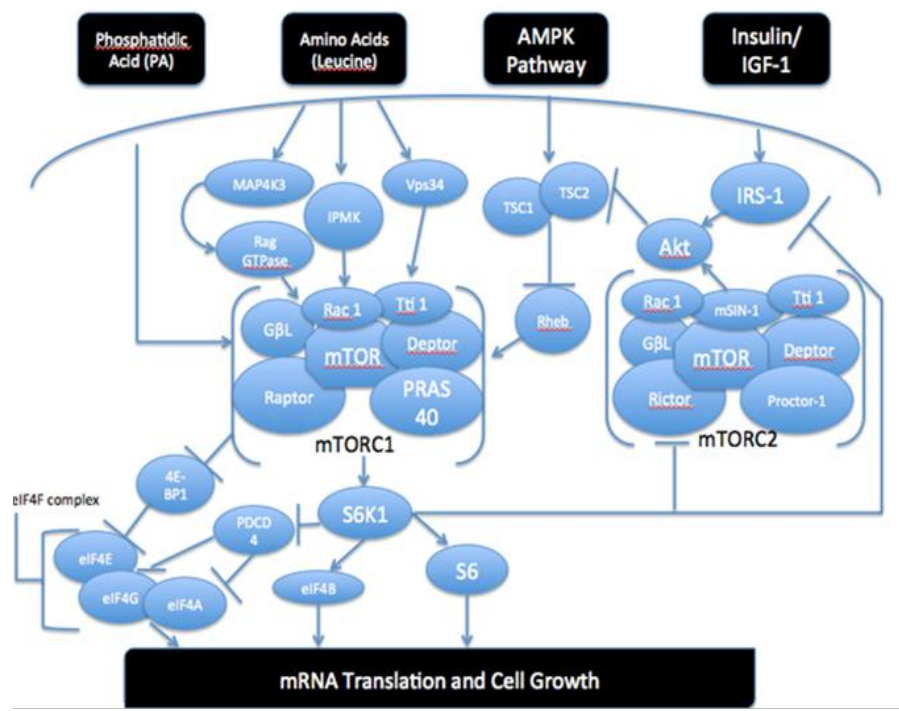

Figure 1: Comprehensive pathway highlighting mTORC1 and mTORC2 complexes and the role that PA, leucine, AMPK pathway and growth the factors, IGF-1 and insulin, play in regulating mRNA translation and cell growth via signal transduction. Figure modified from ${ }^{[20]}$.

Figure 1 also illustrates that the activation of mTOR results in the phosphorylation of other downstream intermediates such as eukaryotic initiation factor 4E-binding protein 1 (4EBP1) and the phosphorylated ribosomal protein S6 (p70S6K) ${ }^{[5,8]}$. 4E-BP1 is an inhibitor of the mRNA cap-binding protein eIF4E ${ }^{[5,8-10]}$. The phosphorylation of 4E-BP1 induces the release

Copy rights: (C2015 Darryn SW. This is an Open access article distributed under the terms of Creative Commons Attribution 4.0 International License. 
of eIF4E and consequently enables the binding with a second initiation factor eIF4G ${ }^{[10-12]}$. The mTOR-mediated phosphorylation of 4E-BP1 results in the recruitment of the eIF4G scaffold onto the 5' strand end of muscle-specific mRNA transcripts. This multi-protein eIF4F complex functions to assist in the binding of mRNA towards the $40 \mathrm{~S}$ ribosomal subunit ${ }^{[8,9]}$, and subsequently initiation protein translation and MPS ${ }^{[11-14]}$

The mTOR pathway also activates p70S6K, which then phosphorylates its effector ribosomal protein S6 (rpS6) that can assist in the enhancement of protein translation from template mRNA $^{[5,8,10,15]}$. The activation of p70S6K, and the subsequent phosphorylation of rpS6, can induce enhanced translation of $\mathrm{mR}$ NAs containing the 5 ' terminal oligopyrimidine tract (5'-TOP) (encoding elongation factors as well as ribosomal proteins. This process leads to increasing protein translation capacity ${ }^{[5,10,11]}$. In addition, the activation of p70S6K assists in bringing rpS6 in closer proximity towards the eIFs (eukaryotic initiation factors) and $\mathrm{mRNA}^{[11]}$. The active p70S6K can also target eIF4B, which favors its binding onto eIF4A. This can increase the helicase activity of eIF4A and provide an additional stimulus for the increasing MPS ${ }^{[12,13]}$. Therefore, the inhibition of this protein kinase within the mTOR pathway would cause a decrease in the rate of MPS ${ }^{[16]}$.

\section{mTORC1 mechanisms of action section:}

mTOR is known to exist in two distinct multi-protein complexes, mTOR complex 1 (mTORC1) and mTOR complex 2 (mTORC2), which are known to differ in their subunit composition, sensitivity to rapamycin, and cell signaling activity ${ }^{[4,17]}$. As seen in Figure 1, both of these complexes contain mTOR and mLST8; however, they are different in that mTORC1 contains the regulatory-associated protein of mTOR (raptor), whereas mTORC2 contains rapamycin-associated protein of mTOR (rictor) ${ }^{[18]}$. With respect to this composition, mTORC1 is highly sensitive to rapamycin whereas mTORC2 is not. mTORC1 activity is regulated by the modulation of the tumor suppressor tuberous sclerosis complex $1 / 2$ activity (TSC $1 / 2)^{[17,18]}$. Figure 1 illustrates this complex to be an active dimer that is purposed to limit mTORC1 signaling through the activity of guanidine trip-phosphatase (GTPase) enzymes. These proteins function to split a phosphate from GTP to yield GDP. Therefore, the TSC $1 / 2$ complex is known to negatively regulate mTORC 1 activity by converting the GTPase protein Rheb (Ras homolog enriched in brain) into its inactive GDP-bound state ${ }^{[17,19]}$. Thus, the GTPase activity of Rheb is highly governed by the TSC $1 / 2$. Moreover, when Rheb is in its active GTP-bound state, it translocates to the lysosome where mTORC1 is activated (Figure 1) ${ }^{[18]}$.

Insulin and IGF-1, are known to repress TSC $1 / 2$ complex activity and allow for the activation of mTORC1 ${ }^{[19]}$. The Akt-induced phosphorylation of mTOR can lead to the inhibition of the tumor sclerosis complex 2 (TSC2) ${ }^{[3]}$. When Akt phosphorylates TSC2, it leads to the cytosolic anchoring protein 143-3 degrading the TSC $1 / 2$ complex $^{[3]}$. This inactivates the GAP activity of TSC2 and represses the hydrolysis of Rheb-GTP, allowing mTORC1 activity to continue ${ }^{[3]}$. Accordingly, Akt can also activate the substrate known asproline-rich Akt substrate of $40 \mathrm{kDa}$ (PRAS40); upon activation PRAS40 translocates away from the Raptor protein located within the mTORC1 complex ${ }^{[20]}$. PRAS40 functions as a repressor of activation by preventing Raptor from recruiting the downstream effectors of
mTORC1 $1^{[21,22]}$. A study observed that the removal of raptor in vitro could minimize the phosphorylation levels of the downstream targets of $\mathrm{mTORC} 1^{[14]}$. Moreover, the raptor protein may be significant in mediating the binding of the substrates 4E-BP1 and $\mathrm{p} 70 \mathrm{~S} 6 \mathrm{~K}$ to the $\mathrm{mTORC} 1$ complex ${ }^{[14]}$.

\section{Effect of leucine on mTORC1 signaling section:}

Leucine mediates mTORC1 signaling through the association with the Rag GTPases, and functions to bind the Raptor subunit of mTORC1 in an amino acid-dependent manner. The Rag GTPases (novel family of four small GTPases, A-D) are known as constitutive heterodimers through which depletion of any Rag heterodimer can inhibit mTORC1 signaling ${ }^{[23]}$. Furthermore, evidence seems to suggest that leucine can promote the binding of Raptor to the Rag heterodimer and Ragulator complex and assist in the translocation of mTORC1 towards the lysosome. Rheb is located at the lysosome; therefore, this would consequently allow for mTORC1 signaling ${ }^{[23,24]}$. The Ragulator complex is known as a trimeric protein complex that is comprised of p14, p18 and MP1 (recently now known as Lyosomal adaptor and MAPK and mTOR activator of LAMTOR 1-3). In the presence of leucine, these Rag GTPases become active with the Ragulator complex and bind $\mathrm{mTORC} 1$ and subsequently translocate this protein onto the lysosome for subsequent integrative activation with GTP-loaded Rheb.

Upon entry into the cell, it has been proposed that leucyl-tRNA (LRS) can directly bind leucine with the Rag GTPases (in particular RagD) which forms the Rag GTPase complex for stimulation of mTORC1 translocation to the lysosome ${ }^{[25]}$. Interestingly, these authors also found that the inhibition of LRS prevented the activation of $\mathrm{mTORC} 1$, even with leucine availability. This highlights the possibility of leucine/LRS activation of GTP activity upon GTPase RagD and subsequent lysosomal recruitment for $\mathrm{mTORC} 1$ activation ${ }^{[24-26]}$. According to the literature, another study proposed that the process of glutaminolysis could allow for the leucine-dependent activation of the RagB heterodimer ${ }^{[27]}$. Glutaminolysis is defined as the conversion of glutamine into alpha-ketoglutarate (alphaKG), which is catalyzed by the enzyme glutamine dehydrogenase (GDH). Furthermore, leucine is allegedly proposed to allosterically assist in the activation of GDH in order to convert glutamine into alpha $\mathrm{KG}$ for the Rag heterodimer loading phase to be activated ${ }^{[27]}$.

Leucine availability may also help facilitate the dissociation of the TSC $1 / 2$ complex from mTOR, which may further raise its activity level ${ }^{[28-30]}$. Furthermore, essential amino acids such as leucine can up-regulate the activity of mTOR as well as its downstream effectors p70S6K and 4E-BP1 in order to maximize intracellular anabolic responses within skeletal muscle. According to the literature, supplementation of leucine is shown to augment the activation of mTOR, its downstream target p70S6K, the phosphorylation of IRS-1, and a decrease in insulin-induced PI3K activity in skeletal muscle of humans ${ }^{[31]}$. It is also known that leucine mediates mTORC1 signaling through the association with the Rag GTPases, which function to bind the raptor subunit of mTORC1 in an amino acid-dependent manner to further promote mTORC1 interaction with activated Rheb-GTP ${ }^{[23]}$. Furthermore, evidence seems to show that leucine ingestion can also mechanistically promote the binding of raptor to the Rag heterodimer and assist in the translocation of mTORC1 towards Rheb, through which this association can 
augment mTORC1 signaling ${ }^{[23]}$.

With respect to nutrient availability, leucine functions as a signaling molecule that can directly activate mTORC1 in an Akt-independent manner. As can be seen in Figure 2, leucine can be transported into the cell by the membrane-bound receptor SLC7A5 (ASC) coupled with SLC3A2 (LAT1/CD98); this process also requires the concomitant active extracellular transportation of glutamine ${ }^{[32,33]}$. This presents clear evidence of the significance behind glutamine for leucine-induced activation of mTORC1. Accordingly, this may explain why glutamine is the most abundant free amino acid within the body, as well as why it is primarily contained within the skeletal muscle ${ }^{[32]}$. Intracellular leucine homeostasis and the rate of cellular uptake is coupled with transporters such as the system L and A transporter (LAT1 and SNAT2, respectively) and highly-correlated with mTORC1 activation $^{[32]}$. Figure 2 illustrates that these transporters primarily function to transport extracellular leucine in exchange for intracellular glutamine. The LAT1 transporter couples with the glycoprotein CD98 alongside the active sodium-linked SNAT2 transporter. SNAT2 functions primarily to regulate glutamine levels within the muscle. The proposed mechanism of action is that LAT1 expels glutamine in exchange for the uptake of leucine, whereas SNAT2 continues to maintain high intracellular glutamine concentrations in order to continue the ability of LAT1 to actively uptake leucine into the cell (Figure 2) ${ }^{[32,34]}$.

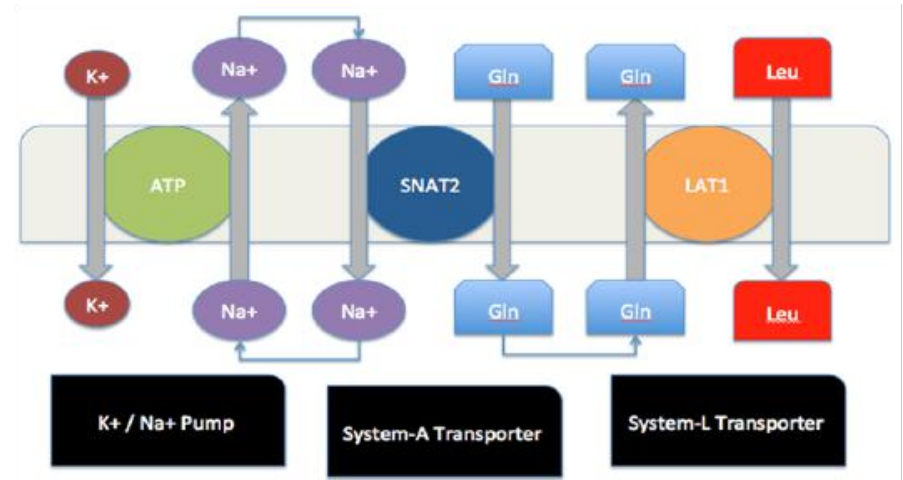

Figure 2: Amino acid transporters for leucine and glutamine, with the coupled SNAT2/LAT1 system. Figure modified from ${ }^{[32]}$.

\section{Effect of phosphatidic acid on mTORC1 signaling section:}

Phosphatidic acid (PA) is suggested to act as an intracellular lipid second messenger which mediates protein signaling activity. PA is also known as a precursor for the biosynthesis of other lipids and is also a major constituent of cell membranes ${ }^{[35]}$. Dietary food sources such as cabbages, radish leaves, and the Mallotus japonicas, a Japanese edible herb, is known to be rich in $\mathrm{PA}^{[36]}$. Several studies have presented observations showing that the stimulation of cells with exogenous administration of PA, or over expression of enzymes that produce PA, can induce increases in mTORC1 activity ${ }^{[12,19,37-39]}$. During in vitro conditions, $\mathrm{PA}$ is also known to associate with the FKBP12-rapamycin-binding (FRB) domain of mTORC1 ${ }^{[40,41]}$. A competition between PA and FKBP12-rapamycin has been noted for FRB binding, which confirms that elevated levels of intracellular PA can activate mTORC1 signaling in this fashion ${ }^{[42,43]}$. As seen in Figure 3, PA is synthesized by various classes of enzymes such as phospholipase D (PLD), lysophosphatidic acid acyltransferases (LPAAT), and diacylglycerol kinases (DAGKs) ${ }^{[42,43]}$. PLD is known to synthesize PA from phosphatidylcho- line (PC), whereas DAGKs synthesize PA from diacylglycerol (DAG) (Figure 3) ${ }^{[44]}$.

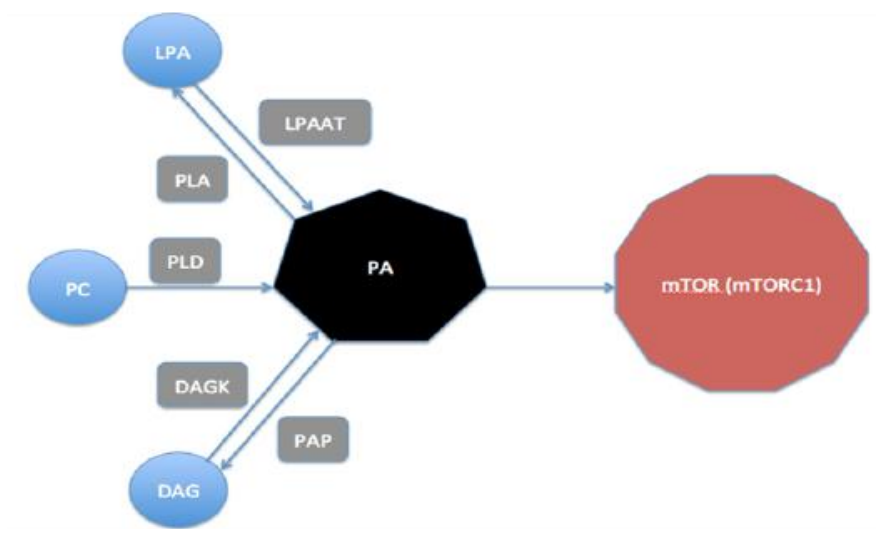

Figure 3: Simplistic overview of the lipids and enzymatic pathways that produce PA and eventually activate mTORC1. Figure modified from ${ }^{[44]}$.

Figure 3 also highlights that DAGK is an enzyme that phosphorylates DAG to produce PA. It has been noted that the over expression of the DAGK-Z isoform can induce mTORC1 signaling, but can be inhibited when the PA binding domain of FRB on mTORC1 is mutated ${ }^{[44]}$. Researchers have found that mechanical stimulation could induce an increase in PA and mTOR signaling due to increases in DAG and DAGK-Z isoform activity ${ }^{[45]}$. This led the authors to suggest that the over expression of DGK-Z may be sufficient to induce MPS, independent of PLD activity. The LPAATs are another potential mediator of PA production and $\mathrm{mTORC} 1$ signaling activity in skeletal muscle (Figure 3), as these enzymes function to catalyze the acylation of LPA in order to form $\mathrm{PA}^{[46]}$. The over expression of the LPAAT enzyme can induce mTORC1 signaling and may highlight potential roles in mediating PA and mTORC1 activity ${ }^{[39]}$.

There are two mammalian isoforms of PLD (PLD 1 and 2), through which PLD1 is known to be a Rheb effector (Figure 4). PLD1 is associated with PA and the TSC $1 / 2$ complex-Rheb complex within the mTORC1 pathway ${ }^{[41]}$. In accordance to the regulation of mTORC1 signaling, it has been shown that the over expression of PLD1 was associated with an increase in mTORC1 activity ${ }^{[42]}$. This was further confirmed by an opposite effect noticed following siRNA-mediated knockdown of PLD1. This suggests that PLD1-derived PA may promote the regulation of mTORC1 signaling. According to researchers, the over expression of PLD1 was found to be associated with a decrease in the expression of the E3 ligase atrophic genes, atrogin-1 and MuRF1, thereby suggesting another mechanistic pathway of convergence ${ }^{[47]}$. Moreover, this over expression of PLD1/PA and down-regulation in the atrophic-related genes may be due to Akt-induced activation by mTORC2 activation ${ }^{[6,40,48]}$. These findings highlight the suggestion that PLD1-derived PA may activate mTORC1 and also mediate the inhibition of proteolysis within the ubiquitin proteasome pathway by activating $\mathrm{mTORC} 2$ and subsequent Akt phosphorylation. Furthermore, PLD1 is suggested to be required for $\mathrm{mTORC} 1$ activity through the interaction with Rheb in a GTP-dependent manner with amino acid availability ${ }^{[49]}$. It is important to note that since PA is a direct activator of mTORC1 activity due to its ability to bind the FRB domain, this interaction is highly contingent upon the state of Rheb ${ }^{[50]}$.

Moreover, as seen in Figure 4, MPS may be induced by increases in PA levels, which may occur through a PLD-de- 
pendent mechanistic pathway ${ }^{[38,44,51]}$ (Figure 4). With respect to the mechanism of action behind mTORC1 activation, it was recently reported that the Ras family of GTPases, RalA, was also required for amino acid induction of mTORC1 activity ${ }^{[52,53]}$. Moreover, RalA is also known to be constitutively associated with PLD1, which consequently demonstrates an importance of PA. Although RalA does not directly activate PLD1, this GTPase protein is known to stimulate the association of PLD1 with ADP-ribosylation factor (ARF) family GTPases. Consequently, this leads to an increase in PLD1 activity ${ }^{[44-56]}$. It has also been suggested that GTP-loaded Rheb also has an association with PLD1 activity, which strengthens the molecular pathways underlying mTORC1 translocation. As illustrated in Figure 4, leucine may also work through a class 3 PI3K receptor known as human vacuolar protein sorting 34 (hVps34), upstream of mTORC1 parallel to the pathway of the Rag GTPase/Ragulator complex ${ }^{[43]}$. This receptor mediates amino acid availability upstream of mTORC1 through which the main product of hVps34 catalysis is $\mathrm{PI} 3 \mathrm{P}^{[43]}$. Furthermore, PI3P is alleged to then bind the PX domains that are present within the signaling proteins such as PLD1. Moreover, this suggests that leucine can stimulate mTORC1 translocation to the lysosome to be activated by mitogen-activated Rheb, which is mediated by the parallel pathways of the hVps34 and the Rag-Ragulator complex (Figure 4) ${ }^{[41]}$.

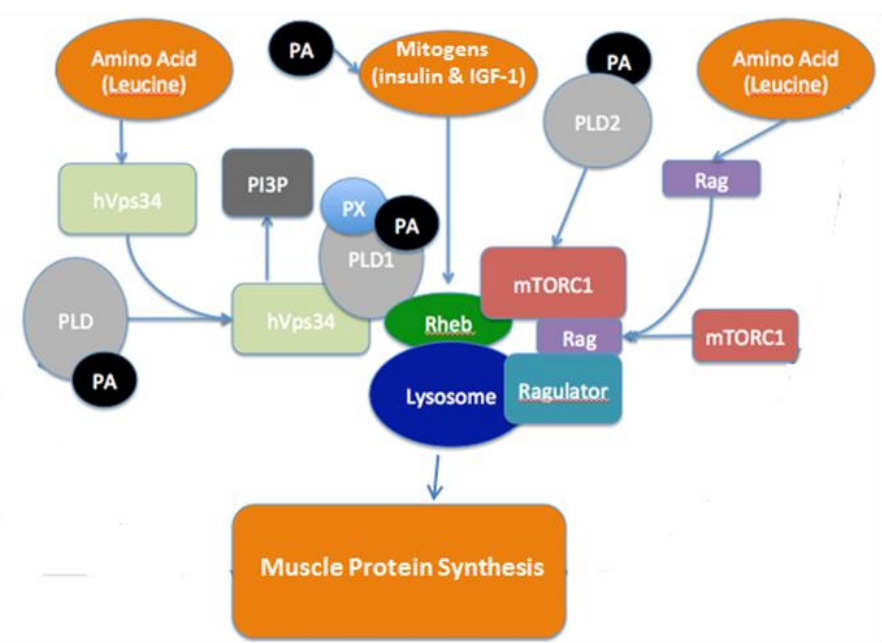

Figure 4: A proposed model highlighting parallel pathways for leucine- and PA-induced activation of mTORC1 and the subsequent effects of MPS.In addition, outside stimuli such as insulin and IGF-1 can up-regulate activity of the upstream efforts of mTOR, which may assist in elevating mTORC1 activity. Figure modified from ${ }^{[41]}$.

Interestingly, another alternative mechanism through which PA allegedly promotes activation of mTORC1 may also be through the ERK signaling pathway ${ }^{[18]}$. Various studies have shown that PA binds activates the Raf protein, an upstream mediator of ERK 1/2. This process then causes ERK 1/2 to phosphorylate its downstream effector, p90RSK, thereby negatively inhibiting TSC $1 / 2$ and increasing mTORC1 activity. Furthermore, the availability of PA to activate ERK $1 / 2$ and mTORC1 may be through the hydrolysis of PA to LPA by phospholipases (such as phospholipase A). This process could then lead to subsequent binding of LPA to endothelial differentiation gene (EDG) receptors (EDG-2) ${ }^{[18]}$. The binding to EDG-2 then leads into the activation of the ERK signaling pathway, which may ultimately up-regulate mTORC1 activity.

\section{Summary}

The intricacy of the complex mTORC1 signaling pathway constitutes a greater need for continual scientific investigation. Several mechanisms of action have been discussed within this review that highlight the converging signals upon mTORC1 such as insulin, IGF-1, leucine, and PA that can lead to increased rate of protein translation necessary for the process of MPS. In contrast to the well-acknowledged PI3K/Akt signaling cascade, recent literature has begun to elucidate the significance of the PLD/PA axis for mTORC1 activation. Recently, there is evidence showing that mTORC1 activation is dependent upon the translocation to the lysosome where Rheb is located. Furthermore, there seems to be a heightened role in levels of PA directly acting upon mTORC1 by the FRB domain. Furthermore, recent results also indicate that the hVps34/PI3P/PLD1 pathway presents a parallel signaling mechanism alongside the well-established Rag/Ragulator complex for ultimately activating mTORC1. However, there is presently a lack of understanding as to how leucine directly interacts with hVps34 and the Rag GTPases. Therefore, further explorations into the roles that leucine and PA have on mTORC1 activity and MPS are warranted.

\section{References}

1. Bodine, S. C., Stitt, T. N., Gonzalez, M., et al. Akt/mTOR pathway is a crucial regulator of skeletal muscle hypertrophy and can prevent muscle atrophy in vivo. (2001) Nature Cell Biol 3(11): 1014-1019.

2. Rasmussen, B.B. Phosphatidic acid: a novel mechanical mechanism for how resistance exercise activates mTORC1 signalling. (2009) J Physiol 587(Pt 14): 3415-3416.

3. Sandri, M. Signaling in muscle atrophy and hypertrophy. (2008) Physiology 23: 160-170.

4. Schiaffino, S., Dyar, K. A., Ciciliot, S., et al. Mechanisms regulating skeletal muscle growth and atrophy. (2013) FEBS J 280(17): 42944314.

5. Zanchi, N. E., Lancha, A. H. Jr. Mechanical stimuli of skeletal muscle: implications on mTOR/p70s6k and protein synthesis. (2008) Eur J ApplPhysiol 102(3): 253-263.

6. Stitt, T. N., Drujan, D., Clarke, B. A., et al. The IGF-1 / PI3K / Akt Pathway Prevents Short Article Expression of Muscle Atrophy-Induced Ubiquitin Ligases by Inhibiting FOXO Transcription Factors. (2004) Mol Cell 14(3): 395-403.

7. Nader, G. A. Molecular determinants of skeletal muscle mass: getting the "AKT" together. (2005) Int J Biochem Cell Biol 37(10): 1985-1996. 8. Bolster, D. R., Jefferson, L. S., Kimball, S. R. Regulation of protein synthesis associated with skeletal muscle hypertrophy by insulin-, amino acid- and exercise-induced signalling. (2004) Proc Nutr Soc 63(2): 351-356.

9. Deldicque, L., Atherton, P., Patel, R., et al. Decrease in Akt/PKB signalling in human skeletal muscle by resistance exercise. (2008) Eur J Appl Physiol 104(1): 57-65.

10. Kubica, N., Bolster, D.R., Farrell, P.A., et al. Resistance exercise increases muscle protein synthesis and translocation of eukaryotic initiation factor $2 \mathrm{~B}$ ? mRNA in a mammalian target of rapamycin-dependent manner. (2005) J. Biol Chem 280(9): 7570-7580.

11. Koopman, R., Zorenc, A.H., Gransier, R.J., et al. Increase in S6k1 phosphorylation in human skeletal muscle following resistance exercise occurs mainly in type II muscle fibers. (2006) Am J Physiol Endocrinol Metab 290(6): E1245-1252.

12. You, J. S., Frey, J. W., Hornberger, T. A. Mechanical stimulation induces mTOR signaling via an ERK-independent mechanism: implications for a direct activation of mTOR by phosphatidic acid. (2012) PloS One 7(10): e47258. 
13. Liu, Y., Vertommen, D., Rider, M.H., et al. Mammalian target of rapamycin-independent S6K1 and 4E-BP1 phosphorylation during contraction in rat skeletal muscle. (2013) Cell Sig 25(9): 1877-1886.

14. Long, X., Lin, Y., Ortiz-Vega, S., et al. Rheb binds and regulates the mTOR kinase. (2005) Curr Biol 15(8): 702-713.

15. Katta, A., Kakarla, S., Wu, M., et al. Altered regulation of contraction-induced Akt/mTOR/p70S6k pathway signaling in skeletal muscle of the obese Zucker rat. (2009) Exp Diabetes Res 2009: 1-9.

16. Hornberger, T. A., Sukhija, K.B., Chien, S. Regulation of mTOR by Mechanically Induced Signaling Events in skeletal muscle. (2006) Cell Cycle 5(13): 1391-1396.

17. Laplante, M., Sabatini, D. M. mTOR Signaling. (2011) Cold Spring Harb Perspect Biol 4(2): 1-3.

18. Winter, J. N., Fox, T. E., Kester, M., et al. Phosphatidic acid mediates activation of mTORC1 through the ERK signaling pathway. (2010) Am J Physiol Cell Physiol, 299(2): C335-344.

19. Jacobs, B.L., Goodman, C.A., Hornberger, T.A. The mechanical activation of mTOR signaling: an emerging role for late endosome/lysosomal targeting. (2014) J Muscle Res Cell Motil 35(1): 11-21.

20. Adegoke, O.A., Abdullahi, A., Tavajohi-Fini, P. mTORC1 and the regulation of skeletal muscle anabolism and mass. (2012) Appl Physiol Nutr Metab 37(3): 395-406.

21. Wang, H., Zhang, Q., Wen, Q., et al. Proline-rich akt substrate of $40 \mathrm{kDa}$ (PRAS40): a novel downstream target of PI3K/Akt signaling pathway. (2012) Cell Sig 24(1): 17-24.

22. Efeyan, A., Zoncu, R., Sabatini, D.M. Amino acids and mTORC1: from lysosomes to disease. (2012) Trends Mol Med 18(9): 524-533.

23. Oshiro, N., Rapley, J., Avruch, J. Amino acids activate mammalian target of rapamycin (mTOR) complex 1 without changing rag GTPase guanyl nucleotide charging. (2014). J Biol Chem 289(5): 2658-2674.

24. Duran, R.V., Hall, M.N. Leucyl-tRNA synthetase: double duty in amino acid sensing. (2012) Cell Res 22(8): 1207-1209.

25. Han, J.M., Jeong, S.J., Park, M.C., et al Leucyl-tRNA synthetaae is an intracellular leucine sensor for the mTORC1-signaling pathway. (2012) Cell 149(2): 410-424.

26. Taylor, P.M. Role of amino acid transporters in amino acid sensing. (2014) Am J Clin Nutr 99(1): 223S-230S.

27. Duran, R.V., Oppliger, W., Robitaille, A.M., et al. Glutaminolysis activates Rag-mTORC1 signaling. (2012) Mol Cell 47(3): 349-358.

28. Browne, G.J., Proud, C.G. Regulation of peptide-chain elongation in mammalian cells. (2002) Eur J Biochem 269(22): 5360-8.

29. Deldicque, L., Theisen, D., Francaux, M. Regulation of mTOR by amino acids and resistance exercise in skeletal muscle. (2005) European Journal of Applied Physiology94(1-2): 1-10.

30. Jefferson, L.,S., Kimball, S.R. Amino acids as regulators of gene expression at the level of mRNA translation. (2003) J Nutr 133(6): 2046S-2051S.

31. Tremblay, F., Krebs, M., Dombrowski, L., et al. Over activation of S6 kinase 1 as a cause of human insulin resistance during increased amino acid availability. (2005) Diabetes 54(9): 2674-2684.

32. Dodd, K.M., Tee, A.R. Leucine and mTORC1: a complex relationship. (2012) Am J Physiol Endocrinol Metab 302(11): E1329-42.

33. Nicklin, P., Bergman, P., Zhang, B., et al. Bidirectional transport of amino acids regulates mTOR and autophagy. (2009) Cell 136(3): 521-34.

34. Baird, F.E., Bett, K.J., Maclean, C., et al. Tertiary active transport of amino acids reconstituted by coexpression of System A and L transporters in Xenopus oocytes. (2009) Am J Physiol Endocrinol Metab 297(3): E822-29.

35. Joy, J.M., Gundermann, D.M., Lowery, R.P., et al. Phosphatidic acid enhances mTOR signaling and resistance exercise induced hypertrophy. (2014) Nutr Metab 11: 29-39.

36. Purpura, M., Jager, R., Joy, J.M., et al. Effect of oral administration of soy-derived phosphatidic acid on concentrations of phosphatidic acid and lyso-phosphatidic acid molecular species in human plasma. (2013) J Int Soc Sports Nutr 10(Suppl 1): P22.
37. Foster, D.A. Phosphatidic acid signaling to mTOR: signals for the survival of human cancer cells. (2009) Biochim Biophys Acta 1791(9): 949-55.

38. O’Neil, T.K., Duffy, L.R., Frey, J.W., et al. The role of phosphoinositide 3-kinase and phosphatidic acid in the regulation of mammalian target of rapamycin following eccentric contractions. (2009) J Physiol 587(14): 3691-3701.

39. Tang, W., Yuan, J., Chen, X., et al. Identification of a novel human lysophosphatidic acid acyl-transferase, LPAAT-theta, which activates mTOR pathway. (2006) J Biochem Mol Biol 39(5): 626-35.

40. Jaafar, R., Zeiller, C., Pirola, L., et al. Phospholipase D regulates myogenic differentiation through the activation of both mTORC1 and mTORC2 complexes. (2011) J Biol Chem 286(25): 22609-21.

41. Yoon, M., Du, G., Backer, J.M., et al. Class III PI-3-kinase activates phospholipase D in an amino acid-sensing mTORC1 pathway. (2011) J Cell Biol 195(3): 435-47.

42. Goodman, C.A., Hornberger, T.A. New roles for Smad signaling and phosphatidic acid in the regulation of skeletal muscle mass. (2014). F1000Prime Reports 6: 20-29.

43. Sun, Y., Chen, J. mTOR signaling: PLD takes center stage. (2008) Cell Cycle 7(20): 3118-3123.

44. Hornberger, T.A. Mechanotransduction and the regulation of mTORC1 signaling in skeletal muscle. (2011) The Int J Biochem Cell Biol 43(9): 1267-1276.

45. You, J.S., Lincoln, H.C., Kim, C.R., et al. The role of diacylglycerol kinase zeta and phosphatidic acid in the mechanical activation of mammalian target of rapamycin (mTOR) signaling and skeletal muscle hypertrophy. (2014) J Biol Chem,289(3): 1551-1563.

46. Lu, B., Jiang, Y.J., Zhou, Y., et al. Cloning and characterization of murine 1-acyl-sn-glycerol 3-phosphate acyltransferases and their regulation by PPARalpha in murine heart. (2005) Biochem J 385(Pt 2): 469-477.

47. Jaafar, R., de Larichaudy, J., Chanon, S., et al. Phospholipase D regulates the size of skeletal muscle cells through the activation of mTOR signaling. (2013) Cell Commun Signal 11: 55.

48. Sandri, M., Sandri, C., Gilbert, A., et al. Foxo transcription factors induce the atrophy-related ubiquitin ligase atrogin- 1 and cause skeletal muscle atrophy. (2004) Cell 117(3): 399-412.

49. Groenewoud, M.J., Zwartkruis, F.J.T. Rheb and Rags come together at the lysosome to activate mTORC1. (2013) Biochem Soc Trans 41(4): 951-955.

50. Buerger, C., DeVries, B.,Stambolic, V. Localization of Rheb to the endomembrane is critical for its signaling function. (2006) Biochem Biophys Res Commun 344(3): 869-880.

51. Hornberger, T.A., Chu, W.K., Mak, Y.W., et al. The role of phospholipase D and phosphatidic acid in the mechanical activation of mTOR signaling in skeletal muscle. (2006b) Proc Natl Acad Sci U S A.103(12): 4741-4746.

52. Maehama, T., Tanaka, M., Nishina, H., et al. RalA functions as an indispensible signal mediator for the nutrient-sensing system. (2008). J Biol Chem 283(50): 35053-35059.

53. Xu, L., Salloum, D., Medlin, P.S., et al. Phospholipase D mediates nutrient input to mammalian target of rapamycin complex 1 (mTORC1). (2011) J Biol Chem 286(29): 25477-25486.

54. Liu, J.Q., Liu, X., Frankel, P., et al. Functional association between Arf and RalA in active phospholipase D complex. (1998) Proc Natl Acad Sci USA 95(7): 3632-3637.

55. Gundermann, D.M., Jager, R., Purpura, M., et al. Soy-derived phosphatidic acid, lysophosphatidic acid and phosphatidylserine are sufficient to induce an increase in mTOR signaling. (2013) J Int Soc Sports Nutr 10(Suppl 1): P7.

56. Hoffman, J.R., Stout, J.R., Williams, D.R., et al. Efficacy of phosphatidic acid ingestion on lean body mass, muscle thickness and strength gains in resistance-trained men. (2012) J Int Soc Sports Nutr 9(1): 47. 